\title{
Trials and Tribulations: mHealth Clinical Trials in the COVID-19 Pandemic
}

\author{
Praveen Indraratna ${ }^{1,2}$, Uzzal Biswas ${ }^{3}$, Jennifer $\mathrm{Yu}^{1,2}{ }^{2}$, Guenter Schreier ${ }^{4}$, Sze-Yuan Ooi ${ }^{1,2}$, \\ Nigel H. Lovell ${ }^{3}$, Stephen J. Redmond ${ }^{3,5}$ \\ 1 Department of Cardiology, Prince of Wales Hospital, Sydney, Australia \\ 2 Prince of Wales Clinical School, The University of New South Wales, Sydney, Australia \\ 3 Graduate School of Biomedical Engineering, The University of New South Wales, Sydney, Australia \\ ${ }^{4}$ AlT Austrian Institute of Technology, Center for Health and Bioresources, Graz, Austria \\ 5 School of Electrical and Electronic Engineering, University College Dublin, Dublin, Ireland
}

\section{Summary}

Introduction: Mobile phone-based interventions in cardiovascular disease are growing in popularity. A randomised control trial (RCT) for a novel smartphone app-based model of care, named TeleClinical Care - Cardiac (TCC-Cardiac), commenced in February 2019, targeted at patients being discharged after care for an acute coronary syndrome or episode of decompensated heart failure. The app was paired to a digital sphygmomanometer, weighing scale and a wearable fitness band, all loaned to the patient, and allowed clinicians to respond to abnormal readings. The onset of the COVID-19 pandemic necessitated several modifications to the trial in order to protect participants from potential exposure to infection. The use of TCC-Cardiac during the pandemic inspired the development of a similar model of care (TCC-COVID), targeted at patients being managed at home with a diagnosis of COVID-19.

Methods: Recruitment for the TCC-Cardiac trial was terminated shortly after the World Health Organization announced COVID-19 as a global pandemic. Telephone follow-up was commenced, in order to protect patients from unnecessary exposure to hospital staff and patients. Equipment was returned or collected by a 'no-contact' method. The TCC-COVID app and model of care had similar functionality to the original TCC-Cardiac app. Participants were enrolled exclusively by remote methods. Oxygen saturation and pulse rate were measured by a pulse oximeter, and symptomatology measured by questionnaire. Measurement results were manually entered into the app and transmitted to an online server for medical staff to review.

Results: A total of 164 patients were involved in the TCC-Cardiac trial, with 102 patients involved after the onset of the pandemic. There were no hospitalisations due to COVID-19 in this cohort. The study was successfully completed, with only three participants lost to follow-up. During the pandemic, 5 of $49(10 \%)$ of patients in the intervention arm were readmitted compared to 12 of $53(23 \%)$ in the control arm. Also, in this period, 28 of $29(97 \%)$ of all clinically significant alerts received by the monitoring team were managed successfully in the outpatient setting, avoiding hospitalisation. Patients found the user experience largely positive, with the average rating for the app being 4.56 out of 5.26 patients have currently been enrolled for TCC-COVID. Recruitment is ongoing. All patients have been safely and effectively monitored, with no major adverse clinical events or technical malfunctions. Patient satisfaction has been high.

Conclusion: The TCC-Cardiac RCT was successfully completed despite the challenges posed by COVID-19. Use of the app had an added benefit during the pandemic as participants could be monitored safely from home. The model of care inspired the development of an app with similar functionality designed for use with patients diagnosed with COVID-19.

\section{Keywords}

Telemedicine, cell phone, mobile applications, smartphone, coronavirus infections

Yearb Med Inform 2021:272-9

http://dx.doi.org/10.1055/s-0041-1726487

\section{Introduction}

Digital health encompasses the use of dedicated remote monitoring units, smartphone applications (henceforth referred to as 'apps'), text messaging services, and wearable devices. Digital health solutions, and in particular mobile-phone based solutions (mHealth), have shown promise in the management of patients with cardiovascular conditions. Patients admitted with acute coronary syndromes or heart failure are prone to readmission soon after discharge. There are several factors that predispose to this. Disease progression or recurrence, which may not be detected in the home environment until the symptoms are severe, is a main causative factor. Several other factors including medication non-compliance, non-attendance at cardiac rehabilitation, and failure to adopt lifestyle changes such as fluid restriction, dietary changes, cigarette smoking cessation, and physical activity may also contribute. An app that could address all of the above factors and thus reduce readmissions to hospital would prove valuable in the modern healthcare setting.
Despite the large number of available mHealth options, the vast majority of interventions are not validated by means of a randomised controlled trial (RCT). mHealth options in cardiovascular disease tend to be divided into two categories - those that provide either education or reminders via text messaging, or those that provide telemonitoring by collecting the results of physical parameters such as weight, pulse and blood pressure. While models of care based on the use of custom-built telemonitoring units have proven benefits in regard to hospitalisation rate and mortality in heart failure (HF) 
patients [1], these stand-alone units can be cumbersome and have limited availability, as they are generally not mass-produced. Apps have the advantage of using the ubiquity and power of the commercial smartphone. A recent meta-analysis demonstrated mobile phone-based interventions were effective in reducing hospitalisations in $\mathrm{HF}$ patients and blood pressure in hypertensive patients [2]. Within this meta-analysis, it was concluded that the most successful apps used data transmission (such as Bluetooth) that minimised the burden on patients to enter and transmit data, and utilised the expertise of the patient's usual healthcare providers, either by alerting them directly to abnormal readings [3] or by having the physician contacted by the research team [4].

The objectives of this article are to (a) describe the implementation of a novel smartphone app within an RCT; (b) describe the impact of the COVID-19 pandemic on the conduct of the trial; and (c) describe how the experience with TeleClinical Care-Cardiac (TCC-Cardiac) guided and informed the development of two telemonitoring programs during the pandemic.

The TCC-Cardiac app, a purpose-built smartphone application, was designed to combine both the educational and telemonitoring aspects in a single app and was planned for an RCT. TCC-Cardiac is a unique and novel model of care for several reasons. Firstly, no app studied in a published RCT has combined both educational and telemonitoring components. Furthermore, RCTs for telemonitoring apps have only been described in the HF population [1, 4-8], making TCC-Cardiac the first telemonitoring app made available to patients with ischemic heart disease in an RCT. TCC-Cardiac is the first telemonitoring RCT for any app in Australian patients with cardiovascular disease, although there are published Australian studies on an educational text messaging intervention [9] and a smartphone app-based model of cardiac rehabilitation [10]. In February 2019, the RCT was commenced at two sites in Sydney, Australia, trialling a model of care that was centred around TCC-Cardiac to supplement standard care, compared to standard care alone. The trial aimed to enrol each participant for six months, with a total enrolment period of 18 months due to rolling recruitment. The onset of the COVID-19 pandemic and the subsequent advice by the Australian government to undertake isolation and social distancing resulted in termination of enrolment and a modification in the running of the trial. Participant follow-up was conducted remotely and gathering of trial data for several secondary endpoints was hindered. These problems affected the vast majority of RCTs across all fields of medicine that were still ongoing when the pandemic began. Despite this, TCC-Cardiac demonstrated its value, particularly during this period.

Furthermore, the promising results of the technology used in the trial inspired the development of two new apps and care models. The first was TCC-COVID, a smartphone app which allowed the transmission of oxygen saturation and symptom data for individuals at home diagnosed with COVID-19, with the aim of providing reassurance and identifying early deterioration in those patients. TCC-COVID was developed, gained ethical approvals and was deployed after thorough security penetration testing in a period of only six weeks. Remote monitoring of patients with COVID-19 via mobile phone technology has been deployed in other healthcare settings. In South Korea, patients with mild infection admitted to a COVID-19 treatment centre recorded their own vital signs and symptoms using a smartphone app. The recordings were integrated into the patient's electronic medical record and were viewed virtually by the medical team who conducted consultations via video-link, thus minimising their direct exposure to the patient [11]. The Cleveland Clinic COVID-19 disease monitoring program also allows for entry of symptoms and pulse oximetry data in patients in home isolation using a mobile app [12]. The Biovitals ${ }^{\circledR}$ Sentinel is currently being used in Singapore and Hong Kong and utilises a Bluetooth-enabled armband for tracking heart rate, blood pressure, temperature and pulse oximetry. The data are transmitted via mobile phone to a central monitoring team [13].

The second new program, TCC-HF, is similar to TCC-Cardiac, but is to be trialled in a single arm implementation study designed for vulnerable HF patients. The rationale of the single arm, non-randomised approach was to offer a solution to all eligible high-risk patients, not just those who would be assigned to the intervention arm. This was an important initiative during the current pandemic as it enabled a telehealth monitoring approach for those vulnerable patients, allowing them to receive remote monitoring without exposing them to the risk of community and hospital cross infections. TCC-HF differs slightly from TCC-Cardiac in that it also incorporates symptomatology questionnaires and pulse oximetry, however the system of data transmission, alert generation and response are largely unchanged from TCC-Cardiac.

\section{The TCC-Cardiac Trial}

\subsection{Methods}

The methods of the original TCC-Cardiac RCT are briefly summarised here. It is anticipated that results of this pilot study will be available later in 2021. 164 in-patients with acute coronary syndrome or HF were randomised 1:1 at the time of discharge, to either receiving the TCC-Cardiac app in addition to standard care, or just standard care alone. To be enrolled, participants must have been adult patients with an admission to hospital for acute coronary syndrome or heart failure, and were capable of providing informed consent and operating the app on their personal smartphone if it was compatible (operating iOS 9 or above or Android 7.0 or above).

The TCC-Cardiac app was released on Apple's App Store and Google's Play Store and was downloaded by the research team members onto the participants' own smartphone (see Figure 1), and a Bluetooth-enabled sphygmomanometer (A\&D Medical, UA-651; Figure 2), weighing scale (A\&D Medical, UC-352; Figure 3), and a wearable fitness wristband (Xiaomi Mi Band II; Figure 4) were supplied and paired. The devices all communicated with the smartphone via Bluetooth low-energy. The devices were tested by twenty volunteer staff members prior to commencement of the trial, and no major problems with data transmission were identified. Participants were encouraged to 
Indraratna et al.

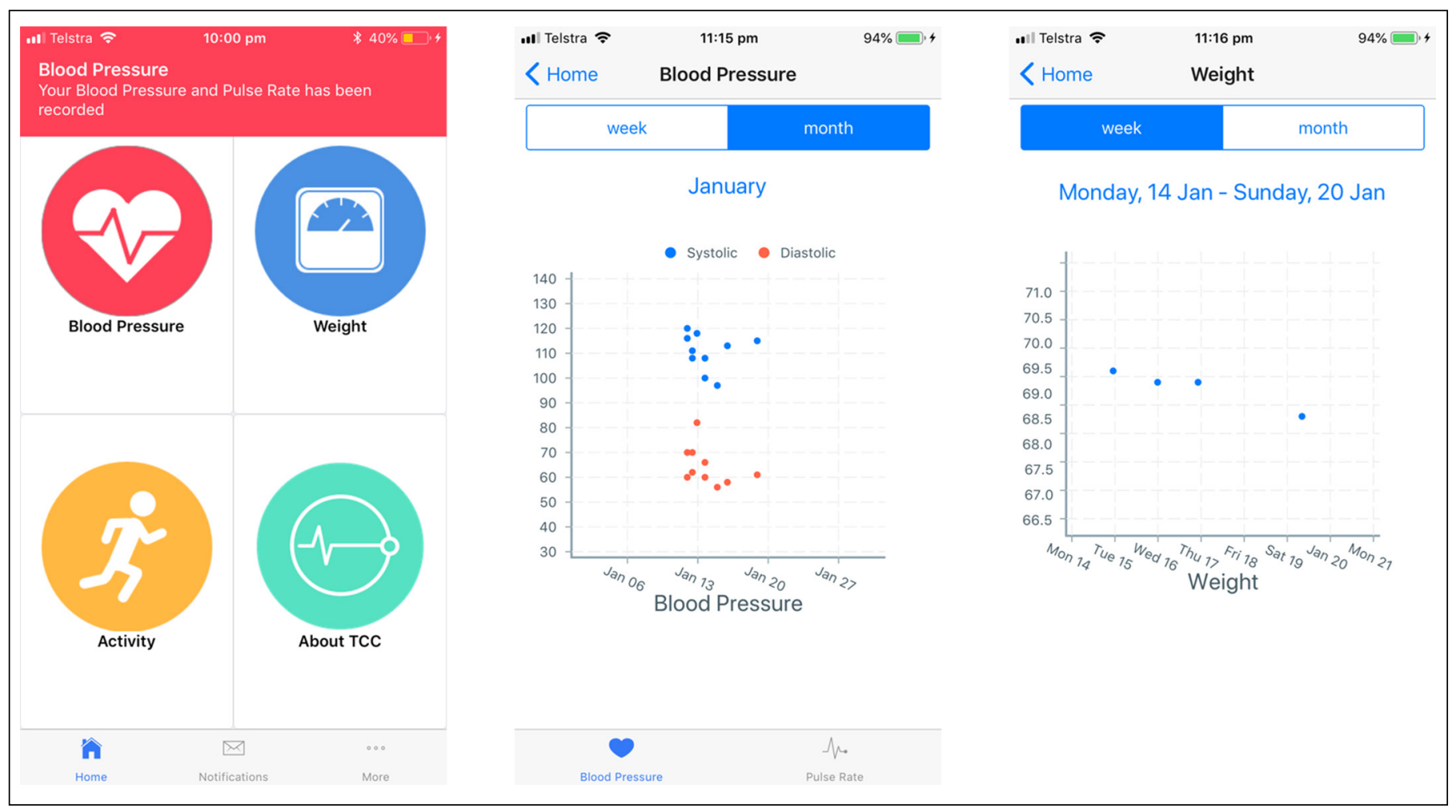

Fig. 1 Screenshots from the TCC-Cardiac app, demonstrating from left to right: the home page, blood pressure graph and weight graph.
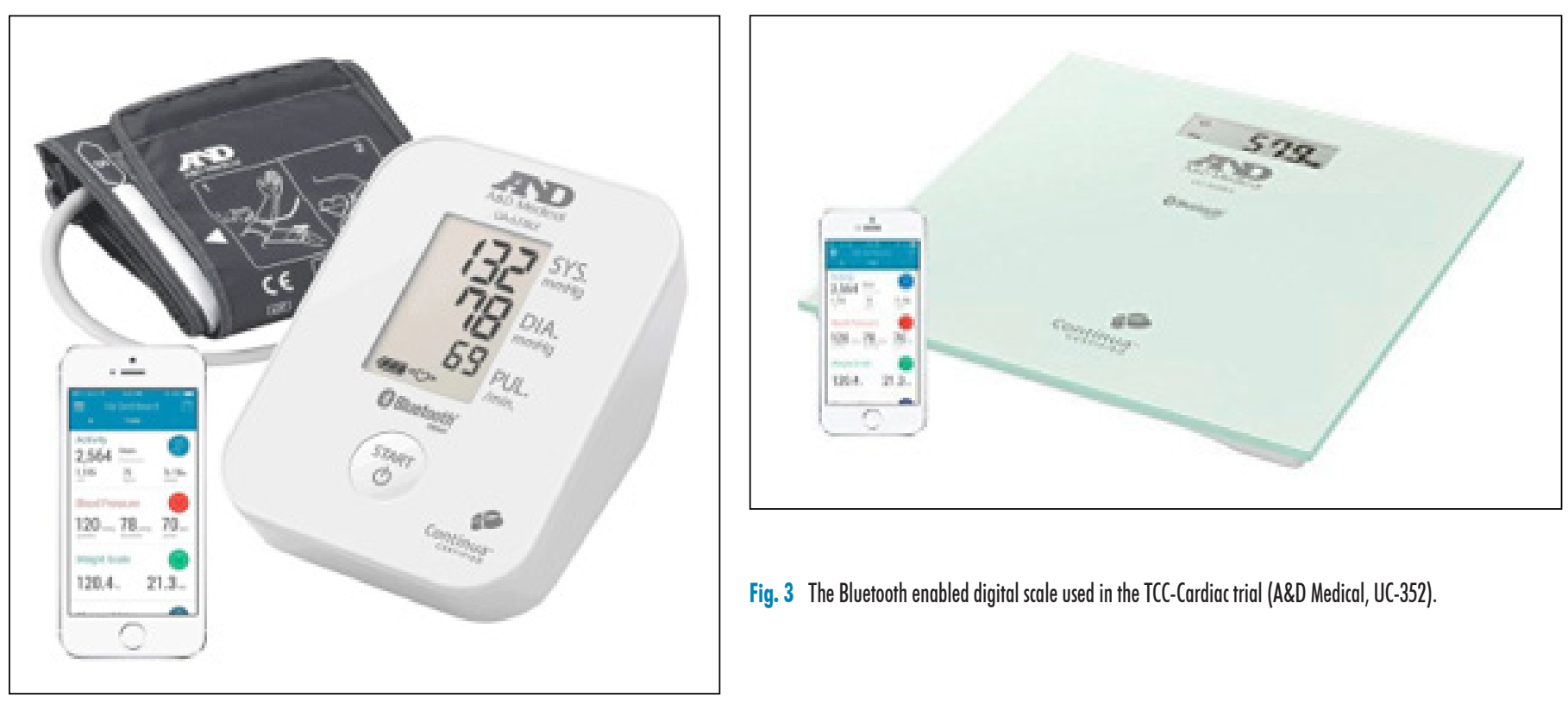

Fig. 3 The Bluetooth enabled digital scale used in the TCC-Cardiac trial (A\&D Medical, UC-352).

Fig. 2 The Bluetooth enabled digital sphygmomanometer used in the TCC-Cardiac trial (A\&D Medical, UA-651). 


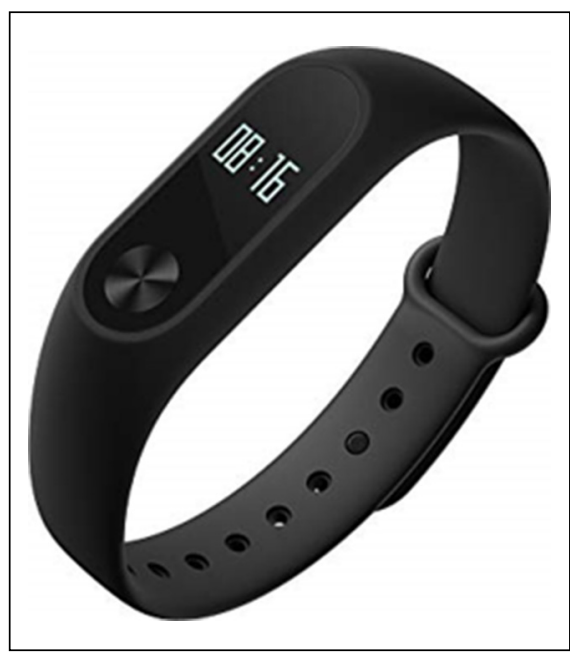

Fig. 4 The wearable fitness band used in the TCC-Cardiac trial (Xiaomi Mi Band II). take their measurements daily. If data were not received after 48 hours, participants were contacted by telephone to ascertain the cause, and assisted with any technical issues. A technical support button was also embedded within the app. The readings from the three peripheral devices were transmitted to the smartphone via Bluetooth and then onwards to a server where it could be explored using a web-based application (called KIOLA, developed at the Austrian Institute of Technology, Figure 5). Aspects of the system including the KIOLA back-end have undergone separate external penetration and acceptance testing by South Eastern Sydney Area Health Service. Pre-defined, customisable thresholds for abnormal values were set by the treating cardiologist at the time of discharge, and these were entered into KIOLA. Readings that subsequently breached any of the thresholds were recognised automatically and a central team of two clinicians were informed immediately by email. Thresholds could be modified at any time during the trial. Alerts were reviewed during business hours and escalated to the patient's treating general practitioner (GP), cardiologist, or HF nurse if necessary. Standard care, including referral to cardiac rehabilitation, was maintained in both control and intervention groups. Participants were followed for six months, at which point they returned to the hospital for an in-person follow-up and returned their equipment. The app also provided educational notifications that encouraged positive lifestyle behaviours, such as healthy eating, physical activity, and smoking cessation.

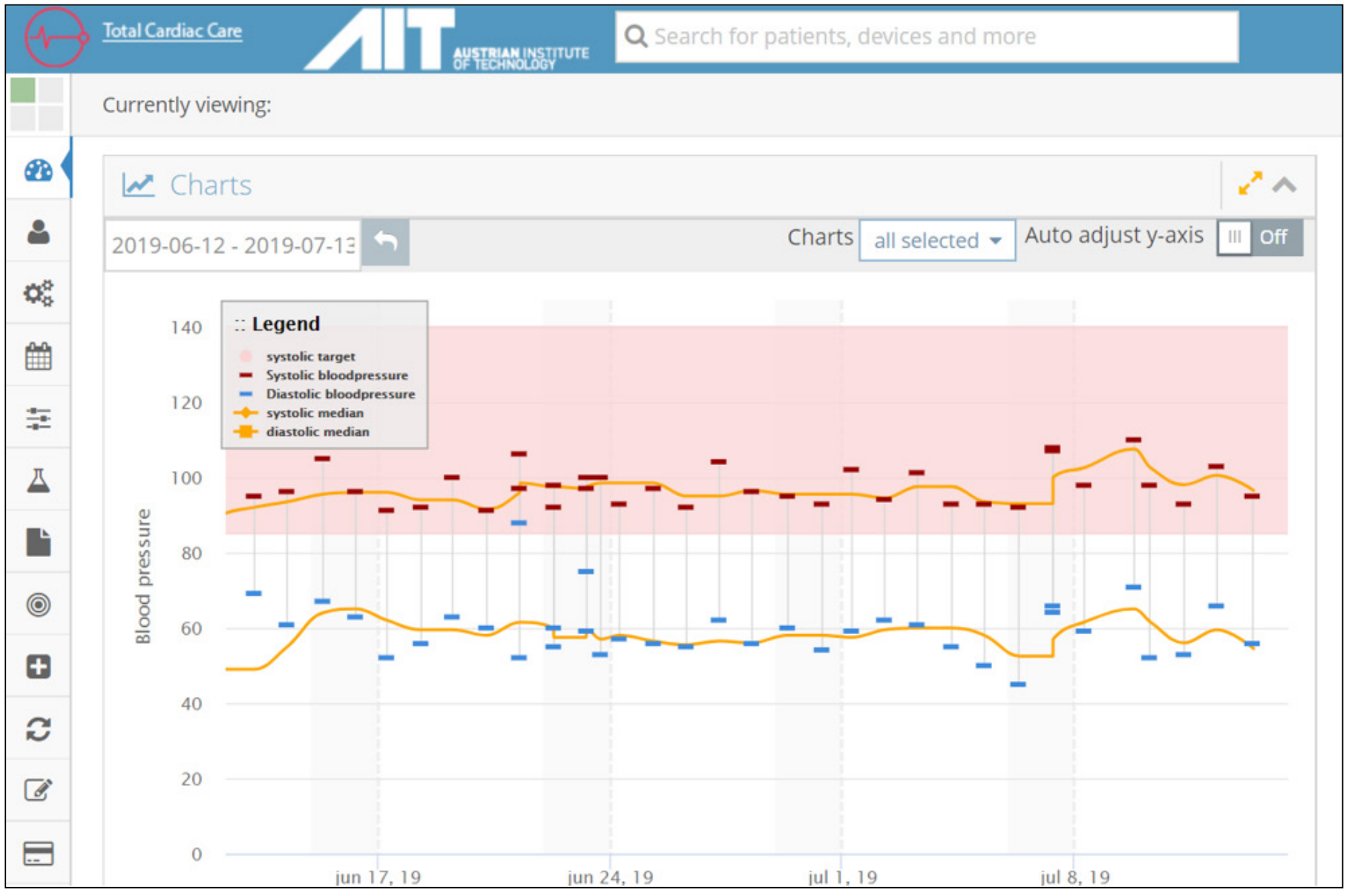

Fig. 5 A screenshot of the KIOLA dashboard used in the TCC-Cardiac trial. The shaded area represents the target systolic blood pressure zone $(85-140 \mathrm{mmHlg}$ in this example). Any reading received outside this zone will trigger an email alert. 
The primary endpoint measure was the incidence of 30-day all-cause readmissions. Secondary endpoints included the 6-month all-cause and cardiac readmissions, length of hospital stay for admissions, cardiac rehabilitation completion rate, low-density lipoprotein (LDL) level, body mass index (BMI), waist circumference, six-minutewalk distance (6MWD), quality of life (as per the EQ-5D questionnaire), and medication compliance (as per the MGL questionnaire). The study was approved by the South Eastern Sydney Local Health District Human Research Ethics Committee (HREC/18/ POWH/91), and the TCC-Cardiac app was registered as a medical device undergoing a clinical trial with the Australian Therapeutic Goods Administration (TGA).

Following completion of the study, the data will be used in the training and validation of a machine-learning model to predict future readmissions.

\subsection{Impact of COVID-19}

In late 2019, a novel coronavirus, (SARSCoV-2), was identified as the cause of an outbreak of acute respiratory illness in Wuhan, China. The disease has been designated COVID-19 (coronavirus disease 2019). Rapid worldwide spread occurred in the months that followed. The World Health Organisation (WHO) declared COVID-19 a global pandemic on March $11^{\text {th }}, 2020$. In response, the Australian government enforced a series of previously unprecedented measures, including mandatory self-isolation, closure of public venues and bans on international travel. Of particular relevance to this study was the early finding of increased mortality among patients infected with the virus who had concomitant cardiovascular disease, which encompassed the entire potential recruitment population for the TCC-Cardiac trial.

Thus, to minimise unnecessary interaction with vulnerable patients, enrolment for the trial was terminated, thirteen months after commencement, and five months prior to the planned conclusion date. It is anticipated that many clinical trials undertaken during this period will have been faced with similar challenges, and interpretation of their findings will need to take this disruption into account.
It was initially hypothesised that the primary endpoint of the trial may be affected, given that 30-day readmission rates may be affected by COVID-19 infections, or by a fall in admissions due to patients avoiding hospital attendance, even in the context of a severe health emergency; this has been previously documented [14]. It was felt, however, that the randomised nature of the trial would mitigate this, as it was anticipated that similar behaviour changes would apply to both groups, and COVID-19 infection rates would be similar between groups. Thus, the trial would continue with the existing participants, but in a modified manner. In-person follow-up, as was scheduled to occur after each participant had been in the trial for six months, was also terminated in order to eliminate COVID-19 infection risk through travel to the hospital and close interaction with the research team. Follow-up occurred via telephone, and the equipment was retrieved using a no-contact drop-off or collection at the hospital or at the patient's home. A user experience questionnaire, designed to evaluate the participants' views on the app itself, was delivered via an online form rather than telephone for simplicity, a change that

Table 1 A description of the modification of the TCC-Cardiac study after the onset of the COVID-19 pandemic

\begin{tabular}{|l|l|l|}
\hline $\begin{array}{l}\text { Recruitment } \\
\begin{array}{l}\text { Patient care } \\
\text { (usual care) }\end{array}\end{array}$ & $\begin{array}{l}\text { Two sites with five-days-per-week recruitment. } \\
\text { Follow up }\end{array}$ & $\begin{array}{l}\text { Cessation of recruitment on March 20, 2020. } \\
\text { participants if they chose to attend. }\end{array}$ \\
$\begin{array}{l}\text { Questionnaires } \\
\text { Participant returned to the hospital after six } \\
\text { months for an in-person follow-up visit. }\end{array}$ & $\begin{array}{l}\text { March 12, 2020. } \\
\text { Follow-up was done via telephone. }\end{array}$ \\
$\begin{array}{l}\text { Return of } \\
\text { equipment }\end{array}$ & $\begin{array}{l}\text { Participant returned to hospital to complete } \\
\text { paper-based questionnaire. }\end{array}$ & $\begin{array}{l}\text { Telephone and online questionnaire options } \\
\text { available. }\end{array}$ \\
$\begin{array}{l}\text { Secondary physical } \\
\text { endpoints (BP, weight, } \\
\text { waist circumference, } \\
\text { LDL, 6MWT) }\end{array}$ & $\begin{array}{l}\text { Participant returned the equipment to staff at } \\
\text { the hospital at the six-month follow-up visit. } \\
\text { months for an in-person follow-up visit. }\end{array}$ & $\begin{array}{l}\text { Returned either by a 'drive-by' or no-contact } \\
\text { pickup at patient's home. }\end{array}$ \\
$\begin{array}{l}\text { Technical support } \\
\text { These endpoints were lost as it was not }\end{array}$ & $\begin{array}{l}\text { considered safe to measure them. } \\
\text { Usually performed via telephone, but the } \\
\text { participant would attend to the hospital if } \\
\text { telephone advice could not fix the problem. }\end{array}$ & $\begin{array}{l}\text { Two participants required home visits for } \\
\text { technical support. Full personal protective } \\
\text { equipment (PPE) and physical distancing } \\
\text { were used. }\end{array}$ \\
\hline
\end{tabular}

was not specific to the COVID-19 pandemic, but rather as a general improvement to streamline remote follow-up. A summary of the trial modifications is included in Table 1.

\subsection{Results}

A total of 164 participants were enrolled ( 81 in the intervention arm and 83 in the control arm). The average age was 62 years in both groups. The proportion of male patients in the intervention and control arms was $80 \%$ and $78 \%$, respectively. $78 \%$ of patients in both groups had a diagnosis of acute coronary syndrome and $22 \%$ in both groups had a diagnosis of $\mathrm{HF}$.

Excluding those lost to follow up, 56 participants finished the study prior to the onset of COVID-19. 43 participants spent $0-3$ months of their time in the trial after the onset of the pandemic, and 59 participants spent 3-6 months in the trial after the onset of the pandemic. Follow up via telephone was safer and more convenient for patients, and only three of 102 patients were unable to be followed-up by telephone. No participants were admitted to hospital due to COVID-19 
during the trial, although data on non-hospitalised mild COVID-19 infections were not collected. From a practical perspective, the modifications made to the TCC-Cardiac trial were largely successful. The major disadvantage was the loss of physical data from the vast majority of these patients, which encompassed several secondary endpoints from the trial such as waist circumference, six-minute walk distance and for patients in the control arm, blood pressure and weight. Nevertheless, data for the primary endpoint (30-day readmissions to hospital) and a major secondary endpoint (6-month readmissions to hospital) are available for all patients except the small number who were lost to follow-up.

Anecdotally, the TCC-Cardiac intervention was incidentally helpful during COVID-19, particularly during the transition to remote consultations. Clinicians across Australia embraced the concept of remote consultations (15), although the obvious drawback in the majority of patients was the lack of measurable physical data such as blood pressure, weight, and pulse rate. For patients using the TCC-Cardiac app, however, the results could be relayed to the GP or cardiologist by the patient, thus providing a more meaningful, comprehensive, and longitudinal virtual assessment of their condition.

Furthermore, the educational notifications provided to the patient by the TCC-Cardiac app were valuable, as patients were not able to receive the educational component of in-person cardiac rehabilitation after it was cancelled. This convenience and unique functionality inspired the design of two further interventions that utilised a similar model of care.

Between the onset of COVID-19 related restrictions in Australia (March 11 $1^{\text {th }}$ 2020) and the end of the trial, the monitoring team received 235 alerts for abnormal physical parameters. 29 clinically-significant alerts were received from a total of 12 patients during this period. 28 of the 29 alerts were successfully managed in the outpatient setting, with only one alert leading to hospitalisation. Examining patients who were enrolled in the study for any duration of time after the onset of the pandemic, there were 5 of 49 patients readmitted in the intervention $\operatorname{arm}(10 \%)$ versus 12 of 53 readmitted in the control arm (23\%). This difference did not reach statistical significance. Results for the entire duration of the trial will be made available in mid-2021.

There was no discontinuation of app use in any patients for technical reasons, including in the 20 elderly patients (age above 70). User satisfaction with the app was high, with the average rating being 4.56 out of 5 . There was no significant difference between the user satisfaction score in those aged 70 or above ( $\mathrm{n}=16$, average rating 4.62 ), compared to those younger than $70(n=50$, average rating 4.54).

\section{Case study:}

The following example demonstrates how use of the TCC-Cardiac system prevented an admission to hospital in an elderly patient who was at risk of severe COVID-19 infection given his age and medical condition.

A 75 year-old man with a history of heart failure transmitted a weight result that indicated a gain of over $2 \mathrm{~kg}$ within one week, suggestive of fluid retention and decompensation of his condition. Subsequently, his heart failure nurse was informed, who visited the patient in full personal protective equipment (PPE) and modified his medication (diuretic) therapy. The patient's condition improved over the next several days, and an admission to hospital was thus avoided. This case study highlights the preventive potential of remote monitoring, both in terms of facilitating early intervention and improved health outcomes, but in reducing healthcare costs through avoidance of unnecessary hospital admission.

\section{TCC-COVID}

\subsection{Methods}

Using a similar framework to the original TCC-Cardiac app, a second app (TCCCOVID) was created and provided to participants who were diagnosed with COVID-19 but did not require hospitalisation and owned a compatible smartphone (operating iOS 9 or above or Android 7.0 or above). The TCCCOVID app was created as a result of a large collaboration between a multidisciplinary team of specialist physicians (cardiology, infectious diseases and respiratory medicine) from three Sydney hospitals and biomedical engineers from the University of New South Wales and the Austrian Institute of Technology. The TCC-COVID app was provided to participants along with a pulse oximeter (Xuzhou Yongkang Electronic Science Technology Ltd; YK-84). As Bluetooth pulse oximeters were not available at the time, due to manufacturing delays of Bluetooth electronic chips in China and Japan related to COVID-19, participants were instructed to measure their oxygen saturation and pulse rate using the pulse oximeter and to manually enter the readings into the TCC-COVID app twice daily. In addition, they would complete a symptom questionnaire daily, which is integrated within the app. Examples of questions are provided in Figure 6 and Figure 7. Unlike the original TCC-Cardiac study, no blood pressure or body weight data is recorded by the TCC-COVID app. Thresholds are defined at the time of recruitment for the oxygen saturation level that would trigger an alert, and this is customisable. They can be changed at any time during the study on the advice of the treating infectious disease physician. Abnormal readings or concerning symptomatology trigger an alert to a centralised monitoring team, who monitor the readings between 8 am and $8 \mathrm{pm}$ and contact the participant if required. The alerts were designed as a three-tier system, with emergent alerts which may trigger immediate hospitalisation, red alerts for urgent scenarios, and yellow alerts for semi-urgent scenarios. Yellow alerts encompassed a pulse of 110-119 beats per minute (bpm) or an oxygen saturation $93-96 \%$. Red alerts were triggered by a pulse of 120-139 bpm, and an oxygen saturation of $90-92 \%$. Emergent alerts were triggered by a pulse of $140 \mathrm{bpm}$ or above, or saturations of $89 \%$ or below. The default thresholds are commensurate with advice offered by the National Institute of Health regarding target oxygen saturation for patients with COVID-19 [16]. Heart rate thresholds were agreed upon by expert consensus in the TCC-COVID planning committee based on published reports of COVID experiences around the world [17]. Yellow and red alerts were also triggered by certain responses within the symptomatology questionnaire, such as shortness of breath and fever, or if the participant volunteered via the app that their condition had worsened. Emer- 


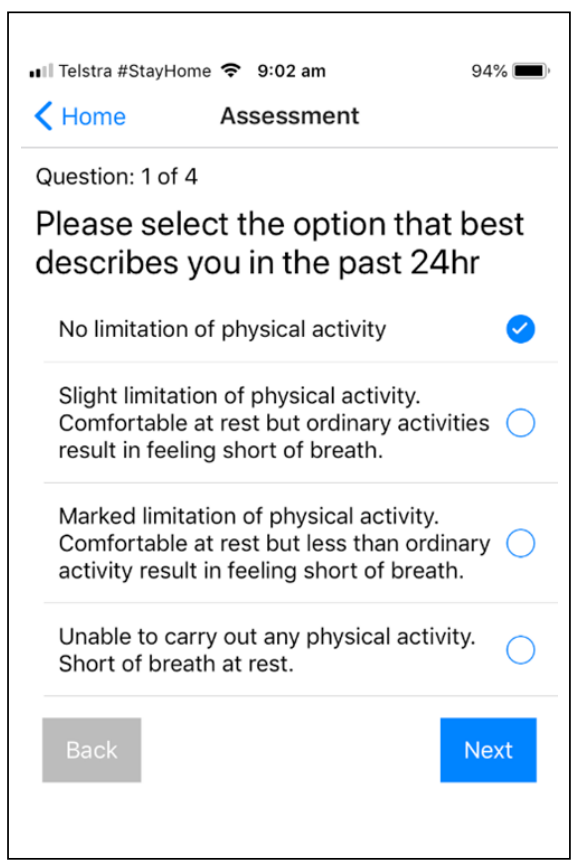

Fig. 6 A screenshot of the physical activity symptomatology question from the TCC-COVID questionnaire.

gent alerts require a response by the monitoring team within 15 minutes, red alerts within 60 minutes and yellow alerts within 180 minutes.

If there is sufficient clinical concern, the participant could be instructed to attend the emergency department via ambulance. Additionally, educational push notifications around COVID-19 and methods to prevent transmission of infection are also provided automatically on a daily basis by the app. The entire enrolment process is conducted remotely, by utilising a combination of email, telephone and video-link for the consent process, thus ensuring participants and research team members do not physically meet, to negate the risk of COVID-19 transmission. Pulse oximeters are delivered by a no-contact delivery method. Whereas in the original TCC-Cardiac study, research team members downloaded the app and demonstrated device use at the patient's bedside, for TCC-COVID instructional videos were created and posted online to educate participants on how to download the app and use the pulse oximeter. The study was approved by the St. Vincent's Hospital Human Research Ethics Committee (2020/ETH01008). Recruitment is currently

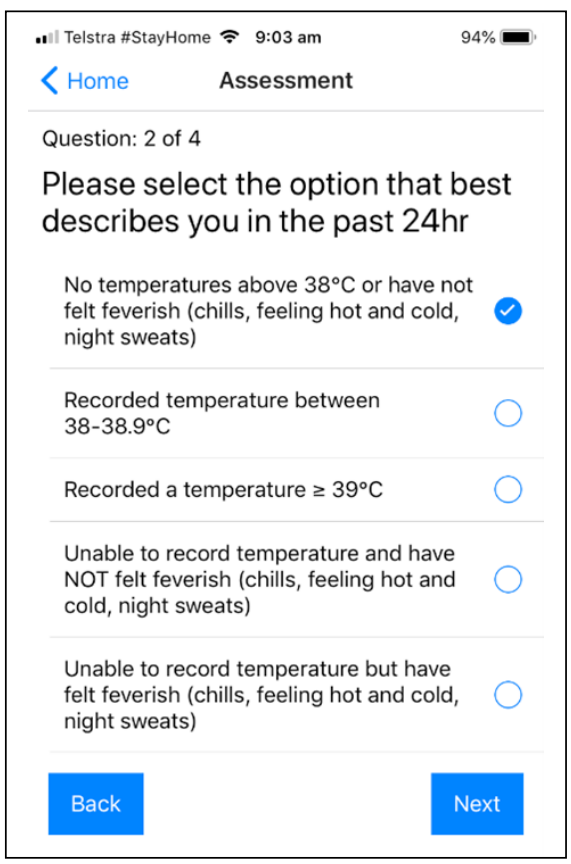

Fig. 7 A screenshot of the fever symptomatology question from the TCC-COVID questionnaire.

ongoing. The system is designated to be used for all patients in the south-eastern Sydney catchment area, comprising a population of approximately 1 million residents. Late in 2020 it was also extended to monitor areas of Melbourne in the state of Victoria.

\subsection{Results}

At the time of writing, 26 participants have been enrolled in the TCC-COVID study at four separate sites. 17 (65\%) patients are male, and the average age is 42 years. All participants have been safely monitored, with no adverse events since enrolment began in May 2020. The app and KIOLA server had functioned as intended, with no major errors. Staff responsible for monitoring patients, all of whom had not previously been involved in an mHealth model of care, reported a steep initial learning curve in adjusting to this method of service delivery. Participant satisfaction has been high, with an average rating of 4.5 stars currently on the basis of preliminary questionnaire data. Detailed results will be available at the conclusion of the study.

\section{Discussion}

COVID-19 has resulted in large changes to society, and also healthcare. In-person medical consultations are becoming less common, as patients avoid hospitals and medical clinics, where they may encounter COVID-19 positive individuals in crowded environments. As such, healthcare needs to adapt. Medicine has been highly reliant on physical examination and manual collection of data from patients, but remote consultations, which may even become the norm, need to be optimised so the patient can derive the most benefit from them. Mobile apps and other technologies that provide data to the clinician can provide a substitute for the standard physical examination. If measurements such as blood pressure and weight are done daily, these may even be superior data to those manually collected in the clinic. This is because longitudinal data is presumed to be more accurate due to a larger sample size of readings, and also trends in the data can be established. In the case of blood pressure, remote measurements can obviate the "whitecoat' effect of increased blood pressure of patients when measured in the clinic environment [18]. For these reasons, clinicians should consider the role of digital health technology in the provision of healthcare to patients both during the pandemic and beyond, either for those whom are diagnosed with COVID-19, or those with other medical conditions for whom attending hospital where clinics may represent an infection risk for the patient.

Across medicine, the conduct of RCTs that commenced prior to the COVID-19 pandemic are likely to be affected. Effects may include termination of recruitment, or in certain cases, termination of the entire trial. Modifying the operation of clinical trials in a way that preserves the original intention of the trial, and the safety of participants, should be considered in these circumstances, particularly in the field of mHealth and digital health, where ongoing participation in the trial may be of significant added benefit.

There are many exciting future possibilities for the management of chronic disease using telemonitoring and wearable devices. Several reviews of wearable and mHealth technologies that would be relevant in the situation of a pandemic have already appeared in the literature [19, 20], but given the immedia- 
cy of the COVID-19 pandemic, the narrative has been centred around future possibilities. In this paper we present specific adaptations of telehealth and app-based solutions in the evolving situation of COVID-19. For example, given the benefits of the TCC-Cardiac model of care, it is planned to distribute a modified version of the app (TCC-HF) to outpatients with HF in the communities served by the hospitals that participated in the original TCC-Cardiac study. There are possibilities for the remote monitoring of many other chronic disease cohorts, such as diabetes mellitus, hypertension and chronic obstructive pulmonary disease (COPD), using similar technologies both during and after the COVID-19 pandemic.

The TCC-COVID program has successfully commenced at three centres in South Eastern Sydney. At the time of writing, the pandemic has fortunately been well controlled in Sydney, and thus fewer participants have been enrolled than expected. However, as a large outbreak occurred in Melbourne, the program has now included a new centre in this city as a result.

\section{Conclusion}

The TCC-Cardiac trial was originally designed to help improve outcomes for patients with acute coronary syndrome and HF. The onset of the COVID-19 pandemic led to early termination of recruitment for this RCT, but the conduct of the study was modified in order to protect already-enrolled participants from attending the hospital. Several secondary endpoints could not be collected as a result. The intervention, although not designed for use during a pandemic, became even more important as readmission to hospital became even less desirable for participants given the potential risk of COVID-19 exposure. The use of this novel digital health innovation during the pandemic inspired the development of two new apps (TCC-COVID and TCC-HF), which would be deployed in a single-arm fashion to assist patients either diagnosed with COVID-19, or outpatients with HF who represent a high-risk population both in terms of hospital readmission, and severe morbidity or mortality from COVID-19 infection.
This experience demonstrated that while COVID-19 may be superficially seen as damaging to the RCT, the trial was adapted, and an innovative response arose as a result. The COVID-19 pandemic has required existing healthcare models to adapt. mHealth and other digital health solutions have never been as critical as they currently are. It is likely that as clinicians and policy-makers become attuned to the benefits of these interventions, their use will continue to increase, even beyond the end of the pandemic.

\section{References}

1. Koehler F, Koehler K, Deckwart O, Prescher S, Wegscheider K, Kirwan BA, et al. Efficacy of telemedical interventional management in patients with heart failure (TIM-HF2): a randomised, controlled, parallel-group, unmasked trial. Lancet 2018;392(10152):1047-57.

2. Indraratna P, Tardo D, Yu J, Delbaere K, Brodie M, Lovell N, et al. Mobile Phone Technologies in the Management of Ischemic Heart Disease, Heart Failure, and Hypertension: Systematic Review and Meta-Analysis. JMIR Mhealth Uhealth 2020;8(7):e16695.

3. Logan AG, Irvine MJ, McIsaac WJ, Tisler A, Rossos PG, Easty A, et al. Effect of home blood pressure telemonitoring with self-care support on uncontrolled systolic hypertension in diabetics. Hypertension 2012;60(1):51-7.

4. Dendale P, De Keulenaer G, Troisfontaines P, Weytjens C, Mullens W, Elegeert I, et al. Effect of a telemonitoring-facilitated collaboration between general practitioner and heart failure clinic on mortality and rehospitalization rates in severe heart failure: the TEMA-HF 1 (TElemonitoring in the MAnagement of Heart Failure) study. Eur J Heart Fail 2012;14(3):333-40.

5. Koehler F, Winkler S, Schieber M, Sechtem U, Stangl K, Böhm M, et al. Impact of remote telemedical management on mortality and hospitalizations in ambulatory patients with chronic heart failure: the telemedical interventional monitoring in heart failure study. Circulation 2011;123(17):1873-80.

6. Scherr D, Kastner P, Kollmann A, Hallas A, Auer J, Krappinger H, et al. Effect of home-based telemonitoring using mobile phone technology on the outcome of heart failure patients after an episode of acute decompensation: randomized controlled trial. J Med Internet Res 2009;11(3):e34.

7. Seto E, Leonard KJ, Cafazzo JA, Barnsley J, Masino C, Ross HJ. Mobile phone-based telemonitoring for heart failure management: a randomized controlled trial. J Med Internet Res 2012;14(1):e31.

8. Vuorinen AL, Leppanen J, Kaijanranta H, Kulju M, Helio T, van Gils M, et al. Use of home telemonitoring to support multidisciplinary care of heart failure patients in Finland: randomized controlled trial. J Med Internet Res 2014;16(12):e282.

9. Chow CK, Redfern J, Hillis GS, Thakkar J, Santo K, Hackett ML, et al. Effect of Lifestyle-Focused Text Messaging on Risk Factor Modification in Patients With Coronary Heart Disease: A Randomized Clinical Trial. JAMA 2015;314(12):1255-63.

10. Varnfield M, Karunanithi M, Lee CK, Honeyman E, Arnold D, Ding H, et al. Smartphone-based home care model improved use of cardiac rehabilitation in postmyocardial infarction patients: results from a randomised controlled trial. Heart 2014;100(22):1770-9.

11. Bae YS, Kim KH, Choi SW, Ko T, Jeong CW, Cho $\mathrm{B}$, et al. Information Technology-Based Management of Clinically Healthy COVID-19 Patients: Lessons From a Living and Treatment Support Center Operated by Seoul National University Hospital. J Med Internet Res 2020;22(6):e19938.

12. Medina M, Babiuch C, Card M, Gavrilescu R, Zafirau W, Boose E, et al. Home monitoring for COVID-19. Cleve Clin J Med 2020 Jun 11.

13. ClinicalTrials.gov [internet]. Using Biovitals ${ }^{\circledR}$ Sentinel to Monitor Disease Progression in Subjects Quarantined for Suspected COVID-192020. Available from: https://clinicaltrials.gov/ct2/show/ NCT04343794

14. Czeisler M, Marynak K, Clarke KEN, Salah Z, Shakya I, Thierry JM, et al. Delay or Avoidance of Medical Care Because of COVID-19-Related Concerns - United States, June 2020. MMWR Morb Mortal Wkly Rep 2020;69(36):1250-7.

15. Fisk M, Livingstone A, Pit SW. Telehealth in the Context of COVID-19: Changing Perspectives in Australia, the United Kingdom, and the United States. J Med Internet Res 2020;22(6):e19264.

16. Shenoy N, Luchtel R, Gulani P. Considerations for target oxygen saturation in COVID-19 patients: are we under-shooting? BMC Med 2020;18(1):260.

17. Chen T, Wu D, Chen H, Yan W, Yang D, Chen G, et al. Clinical characteristics of 113 deceased patients with coronavirus disease 2019: retrospective study. BMJ 2020;368:m1091.

18. Hall WD. Pitfalls in the diagnosis and management of systolic hypertension. South Med J. 2000;93(3):256-9.

19. Adans-Dester CP, Bamberg S, Bertacchi FP, Caulfield B, Chappie K, Demarchi D, et al. Can mHealth Technology Help Mitigate the Effects of the COVID-19 Pandemic? IEEE Open J Eng Med Biol 2020;1:243-8.

20. Ding XR, Clifton D, Ji N, Lovell NH, Bonato P, Chen W, et al. Wearable Sensing and Telehealth Technology with Potential Applications in the Coronavirus Pandemic. IEEE Rev Biomed Eng 2021;14:48-70.

\section{Correspondence to:}

Dr. Praveen Indraratna

Department of Radiology

St. Paul's Hospital

1081 Burrard Street

Vancouver, V6Z 1Y6, Canada

Tel: +16047711839

Fax: + 16048068283

E-mail: praveen@unsw.edu.au 